\title{
Variation of insect attracting odor in endophytic Epichloë fungi: Phylogenetic constrains versus host influence
}

\section{Journal Article}

Author(s):

Steinebrunner, Fabrizio; Schiestl, Florian P.; Leuchtmann, Adrian

Publication date:

2008

Permanent link:

https://doi.org/10.3929/ethz-b-000011458

Rights / license:

In Copyright - Non-Commercial Use Permitted

Originally published in:

Journal of Chemical Ecology 34(6), https://doi.org/10.1007/s10886-008-9476-5 


\title{
Variation of Insect Attracting Odor in Endophytic Epichlö̈ Fungi: Phylogenetic Constrains Versus Host Influence
}

\author{
Fabrizio Steinebrunner • Florian P. Schiestl • \\ Adrian Leuchtmann
}

Received: 15 January 2008 /Revised: 26 March 2008 / Accepted: 3 April 2008 / Published online: 8 May 2008

(C) Springer Science + Business Media, LLC 2008

\begin{abstract}
Odor is a key trait for pollinator attraction in flowering plants, and many studies have investigated odor evolution in the light of pollinator selection by emphasizing the importance of the plant phylogenetic history. By contrast, little is known on the evolution of odors in fungus-insect interactions. In this study, profiles of three volatile compounds that are emitted by grass-inhabiting Epichloë fungi (Clavicipitaceae, Ascomycota) and that have a confirmed or likely role in the attraction of gamete-transferring Botanophila flies were investigated. We collected headspace samples from stromata of six European Epichloë species (including various host races) that originated from different locations in Switzerland, France, Poland, and UK for conducting gas chromatography analyses. Odor profiles exhibited considerable variation, but profiles of most species overlapped and did not discriminate at the species level. The exception was Epichloë festucae, which had a profile dominated by methyl (Z)-3-methyldodec-2-enoate. Based on an Epichloë phylogeny, there was some hierarchical structuring regarding levels of chokol $\mathrm{K}$, another confirmed Botanophila attractant. However, patterns of odor profiles appeared to be largely dependant on particular Epichlö̈-host associations. The observed variation may be the result of complex selective pressures imposed by Botanophila gametic vectors, local environment, and mycoparasites.
\end{abstract}

F. Steinebrunner $\cdot$ A. Leuchtmann $(\triangle \nabla)$

Plant Ecological Genetics, Institute of Integrative Biology (IBZ),

ETH Zürich,

8092 Zürich, Switzerland

e-mail: adrian.leuchtmann@env.ethz.ch

\section{F. P. Schiestl}

Institute of Systematic Botany, University of Zürich,

Zollikerstrasse 107 ,

8008 Zürich, Switzerland
Keywords Chokol $\mathrm{K} \cdot \operatorname{Methyl}(Z)-3$-methyldodec2-enoate $\cdot$ Odor communication $\cdot$ Botanophila . Epichloë endophytes

\section{Introduction}

Odor is a key component of many sessile organisms in communicating with other organisms in an ecosystem. It may be important, alone or in concert with visual stimuli, for the attraction of insects that are involved in pollination or dispersal by eliciting searching, alighting, and feeding (Knudsen 2002; Plepys et al. 2002). Even though pollinator attraction is viewed as the primary function of odor, other functions that include plant defense or protection against abiotic stresses have been documented (Pellmyr and Thien 1986; Dudareva et al. 2006). While odor has received much attention in angiosperms, only a few studies have focused on odors of fungi and even fewer on the role of odor in fungal-insect interactions (Hedlund et al. 1995; Raguso and Roy 1998; Guevara et al. 2000). Recently, the biological function of volatiles for sexual reproduction of Epichloë fungi has been unraveled (Schiestl et al. 2006; Steinebrunner et al. 2008a).

Epichloë fungi (Clavicipitaceae, Ascomycota) are endophytes of pooid grasses with which they form constitutive, life-long symbioses. Infections are symptomless during vegetative growth of the host. Only at the flowering stage does the fungus produce external fruiting structures (stromata) on grass inflorescences. Epichloë is self-incompatible and has to be fertilized by gametes of the opposite mating type for sexual reproduction. Gametes (spermatia) are transferred by specialized flies of the genus Botanophila that feed and develop on fungal stromata (Bultman et al. 1995). Immediately after oviposition, the flies actively 
fertilize the stroma, which involves spreading of viable spermatia containing feces over the stroma surface (Bultman et al. 1998). Thus, fly activity is indispensable for the formation of sexual ascospores that are wind dispersed and may infect new host grasses. Conversely, Botanophila larvae develop on the stroma and rely on the growing fungal tissue as a food source. In general, the Epichlö-Botanophila association is a balanced mutualism for which overexploitation by Botanophila larvae does not occur (Bultman et al. 2000). However, if the conditions are conducive, the mutualism may be disrupted to form an interaction of simple foraging by fly larvae (Rao and Baumann 2004).

The phylogeny of genus Epichloë based on multiple gene sequences is well established (Schardl et al. 1997; Craven et al. 2001). Epichloë comprises ten species, which have been circumscribed as distinct mating populations with one exception (Schardl et al. 1997). The species differ in host range and are restricted to either Eurasia or North America, following the native distribution of the host grasses (Leuchtmann 2003). A recent study of Epichlö̈associated Botanophila revealed that at least six distinct fly taxa are involved in gamete transfer in Europe and North America (Leuchtmann 2007). While phylogenetic analyses indicated that seven Epichloë species coevolved by common descent with grass hosts (Schardl et al. 1997), comparison of molecular phylogenetic relationships of Botanophila with the associated Epichloë hosts did not suggest co-evolution of fungus and fly (Leuchtmann 2007).

Odor communication between Epichloe and the Botanophila flies is based on a relatively simple mechanism that involves two system specific volatiles, namely, chokol $\mathrm{K}$ and methyl (Z)-3-methyldodec-2-enoate (MME; Schiestl et al. 2006; Steinebrunner et al. 2008a). These compounds (and perhaps others) are assumed to be key traits responsible for Botanophila attraction and are likely adaptive for Epichloë and possibly Botanophila flies. In addition, chokol $\mathrm{K}$ is fungitoxic (Koshino et al. 1989) and has been shown to reduce spore germination of Epichlö mycoparasitic fungi (Steinebrunner et al. 2008b). Thus, the production of chokol K and MME in Epichloë may be the result of complex selection pressures.

In plant-insect interactions, odor profiles are often species specific (Levin et al. 2001; Grison-Pige et al. 2002; Knudsen et al. 2006), which potentially enables pollinators to recognize a particular plant species. Likewise, in the Epichloë system, species-specific odor profiles may allow for specific Botanophila attraction and, thus, could maximize successful cross-fertilization. Among three Epichloë species, differences in the production of chokol $\mathrm{K}$ and $\mathrm{MME}$ have been reported (Steinebrunner et al. 2008a). However, regarding Botanophila flies, there is little empirical evidence for host fidelity. With one exception, larval catches from Epichloë stromata indicate that Botanophila species are typically associated with several Epichloë species (Leuchtmann 2007). Similarly, a common Botanophila species did not differentiate between synthetic odor blends that imitate different Epichloë species offered in traps but visited all traps at equal rates (Steinebrunner et al. 2008a). On the other hand, an earlier study that examined gamete mixtures in the fly feces suggested that flies predominantly visited one host while visiting others less frequently (Bultman and Leuchtmann 2003). Therefore, Botanophila flies may display "flower" constancy, a tendency to visit stromata of one species repeatedly while ignoring suitable stromata of another Epichloë species.

A given odor profile may not only be viewed as result of selective biotic forces acting on its producer but may simply be a by-product of metabolic processes, or have remained, although the adaptive context has changed (Levin et al. 2003; Raguso et al. 2003). Therefore, interpretation of odor profiles should be made in a phylogenetic context. A further complication in studying odor in endophytic Epichloë is that its production may be modulated by the host grass, the Epichlö genotype, and/or the interaction of the two.

In the present study, we examined Epichloë odor production at species and at host race levels. We relied on the analysis of stromata emitted, previously described compounds (or its variants) by using headspace sampling. The following questions were asked: (1) How variable are odor profiles among Epichloë species? Are the profiles species specific? Is there evidence for fluctuations among years? (2) Are odor data phylogenetically informative so that they could be used to reconstruct phylogenetic relationships?

Our study is the first describing variation of insect attracting odor in Epichloe fungi and offering insight into phylogenetic patterns of odor production on a genus-wide scale.

\section{Methods and Materials}

Study Taxa and Sampling Plants infected by Epichloë fungi originated from various locations in Switzerland, France, Poland, UK, and USA and had been maintained for up to $14 \mathrm{yr}$ in pots at an experimental garden in Zürich, Switzerland. In total, 14 different host plant-endophyte associations represented by 1-24 genetically distinct accessions were included in the study (Table 1). Before and during the experiment, plants were watered regularly and received liquid fertilizer once a week. To induce stromata formation for odor collection, potted plants were vernalized outside during winter. The following spring when stromata emerged (which usually coincides with host flowering), plants were moved into the laboratory, and volatiles emitted 
Table 1 List of Epichloë associations used in the present study with number of accessions (genotypes) and geographic origin

\begin{tabular}{lllcll}
\hline Fungal species & Host species & Abbreviation & No. of accessions & Geographic origin ${ }^{\text {a }}$ & Country \\
\hline E. baconii & Agrostis tenuis & Eba-At & 4 & ZH & Switzerland \\
E. baconii & Calamagrostis villosa & Eba-Cv & 3 & GR & Switzerland \\
E. bromicola & Bromus erectus & Eb-Be & 17 & SH, AG, LU, Vesancy & Switzerland, France \\
E. clarkii & Holcus lanatus & Ec-Hl & 8 & VD & Switzerland \\
E. festucae & Festuca rubra & Ef-Fr & 6 & VD, VS, Vesancy, Lexington & Switzerland, France, USA \\
E. sylvatica & Brachypodium sylvaticum & Es-Bs & 8 & ZH & Switzerland \\
E. typhina & Anthoxanthum odoratum & Et-Ao & 3 & VD, TI & Switzerland \\
E. typhina & Dactylis glomerata & Et-Dg & 24 & VD, ZH, SH, Vesancy & Switzerland, France \\
E. typhina & Brachypodium pinnatum & Et-Bp & 7 & VD, ZH & Switzerland \\
E. typhina & Phleum pratense & Et-Php & 1 & Cardiff & UK \\
E. typhina & Poa nemoralis & Et-Pn & 5 & VD, ZH & Switzerland \\
E. typhina & Poa pratensis & Et-Pp & 3 & ZH, VS & Switzerland \\
E. typhina & Poa trivialis & Et-Pt & 2 & ZH & Switzerland \\
E. typhina & Puccinellia distans & Et-Pd & 2 & Gniebnia, Janikowo & Poland
\end{tabular}

${ }^{\text {a }}$ Swiss canton ( $A G$ Aargau, GR Grison, $L U$ Luzern, SH Schaffhausen, TI Ticino, VD Vaud, VS Valais, ZH Zürich) or town if outside Switzerland

from the stromata were sampled with headspace sorption within the first $3 \mathrm{~d}$ after emergence. Sequential measurements on stromata from Epichloë typhina infecting Phleum pratense at days $1,3,6,10,13,15,20$, and 23 after stroma emergence demonstrated that volatile production did not decrease considerably until day 3 (Fig. 1) suggesting that our sampling provided an accurate measure for maximum odor production. To estimate consistency of odor emission among years, odor was sampled from a representative subset of plants that included 15 accessions and 7 host grasses in 2 consecutive years (2005 and 2006).

Collection of Fungal Volatiles To collect volatiles, stromata-bearing tillers were covered individually with polyethylene terephtalate bags (Nalo ${ }^{\circledR}$, Kalle $\mathrm{GmbH}$, Germany) and the air pulled out by a vacuum pump (SCK Inc.) at a rate of approximately $100 \mathrm{ml} / \mathrm{min}$. Volatiles were trapped on custom-made micropipette filters containing $5 \mathrm{mg}$ of Porapak Q (Mesh size 80/100, Alltech Associates Inc., USA; Salzmann et al. 2006). If available, two to four stromata per plant were sampled individually, except for some plants where only one stroma-bearing tiller emerged. Before sampling, the Porapak Q adsorbent was cleaned with $100 \mu \mathrm{l}$ of dichlormethane and $100 \mu \mathrm{l}$ of hexane (Merck, Uvasol). Blank air samples were collected to check for background contaminants. After a sampling period of $24 \mathrm{hr}$ (including day and night at room temperature), adsorbed volatiles were eluted from Porapak Q with $50 \mu \mathrm{l}$ of a mixture of hexane/acetone (9:1; Merck), sealed in glass vials, and stored at $-20^{\circ} \mathrm{C}$. Headspace sorption is the method of choice for collecting compounds of medium to high volatility that are produced de novo and emitted immediately, as those from Epichlö stromata examined here, and relatively long sampling periods were necessary to collect sufficiently concentrated samples. Interval sampling of the key volatiles chokol $\mathrm{K}$ and MME did not show differences in emission between day and night (Steinebrunner, unpublished data), thus suggesting continuous odor production. Nevertheless, flies are active only during day, preferably at warm and sunny conditions between 10 A.M. and 4 P.M.

In vitro production of volatiles was examined in isolates of Epichloë grown on potato dextrose agar (Difco) in Petri dishes (Steinebrunner et al. 2008b). For collecting volatiles, Petri dishes with 2-wk-old colonies were placed in polyethylene terephtalate bags $\left(\mathrm{Nalo}^{\circledR}\right.$, Kalle $\left.\mathrm{GmbH}\right)$, then

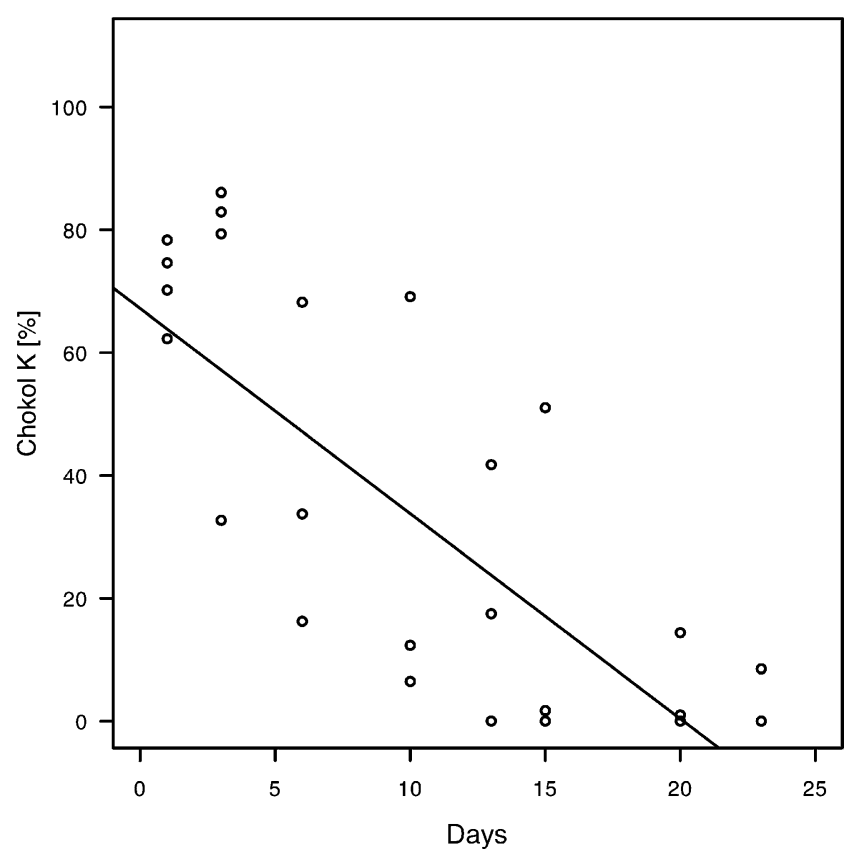

Fig. 1 Scatterplot of repeated headspace measurements from Epichlö typhina stromata on Phleum pratense (Et-Php) at days 1, 3, 6, 10, 13, 15,20 , and 23 after stromata emergence 
the cover was lifted, and the air above the growing Epichloe cultures inside the bag was sampled as described above.

Quantitative GC Analyses and GC-Mass Spectrometry For quantitative analysis, $100 \mathrm{ng}$ of $n$-octadecane were added to samples as an internal standard. One microliter of each sample was injected splitless into a gas chromatograph (GC; Agilent $6890 \mathrm{~N}$ ) equipped with an HP5 column (5\%phenyl-metylpolysiloxane, $30 \mathrm{~m} \times 0.32 \mathrm{~mm}$ diameter $\times$ $0.25 \mu \mathrm{m}$ film thickness) and a flame ionization detector. The column was temperature-programmed at $50^{\circ} \mathrm{C}$ for $1 \mathrm{~min}$, followed by a temperature increase to $300^{\circ} \mathrm{C}$ at a rate of $10^{\circ} \mathrm{C} \mathrm{min}^{-1}$, with a final hold of $10 \mathrm{~min}$ at $300^{\circ} \mathrm{C}$. The carrier gas was hydrogen (Carbagas, local distributor), and the GC was operated at constant flow $\left(2 \mathrm{ml} \mathrm{min}^{-1}\right)$. Data acquisition and data analysis were done by using the Agilent Chemstation software package (Agilent Technologies, Palo Alto, CA, USA).

Previously known compounds (chokol $\mathrm{K}$ and MME) were identified by retention time comparison with authentic standards (Schiestl et al. 2006; Steinebrunner et al. 2008a). Identification of unknown compounds was done by using GC/mass spectrometry (MS) (HP G 1800 A, Hewlett Packard Inc., Palo Alto, CA, USA) with the operating conditions as described above, following tentative identification of the mass spectra on the basis of the NIST database information. To calculate absolute amounts, the internal standard method proposed by Schomburg (1990) was used.

Phylogenetic Analysis To establish a possible link between phylogeny of Epichloë taxa and the observed odor profiles, a maximum likelihood (ML) tree based on variations of intron sequences of $\beta$-tubulin (tub2) of representative strains of European Epichloë species plus the American outgroup taxon E. brachyelytri was constructed. The sequences used have been published in Craven et al. (2001) and were retrieved from GenBank (National Center for Biotechnology Information, Bethesda, MD, USA; http://www.ncbi.nlm.nih.gov/) with one exception. E. typhina from Puccinellia distans $(E t-P d)$ was newly sequenced in the course of this study, following the methods described in Craven et al. (2001), and submitted to GenBank (accession number EU375739). Aligned sequences were analyzed with PAUP $* 4.0 \mathrm{~b} 10$ (Swofford 2003) employing ML and maximum parsimony analyses. ModelTest v.3.7 (Posada and Crandall 1998) was used to select the best fitting model $(\mathrm{K} 80+\mathrm{G})$ for general time reversible $\mathrm{ML}$ analysis by using the tree bisection and reconnection branch swapping algorithm. Odor profiles from in vitro and in vivo results, including three compounds [chokol K, MME, and methyl 2,4,8-trimethylundecanoate (MTE)], plus a group of unknown diterpenes were then mapped into the ML phylogenetic tree. Phylogenetic reconstruction was done separately with odor data, but as expected, the resulting tree was poorly resolved due to the relatively small data set.

Statistical Analyses All comparisons of odor compounds from different Epichloë species and host races are based on relative amounts. We use relative values for technical reasons to provide a more reliable measure for comparison. Absolute values from samples that are not simultaneously processed are difficult to compare due to variations in absorption and elution of filters. Relative values of single compounds were calculated by dividing the amount of each compound by the sum of all other compounds in the headspace (excluding the solvent peak). Homogeneity of variances of data was tested with Levene's test. If no adequate transformation were found, data were analyzed by using the nonparametric Kruskal-Wallis test. Principal component analysis based on the three investigated compounds (chokol K, MME, and MTE) was used to illustrate the relationship among species. Sequential odor emission measurements in E. typhina from Phleum pratense (Et-Php) were analyzed with one-way analysis of variance based on log-transformed values. For comparisons of odor emission among years of selected genotypes, the 95\% confidence interval $(95 \% \mathrm{CI})$, based on the differences of the means of the $2 \mathrm{yr}$, was calculated. If zero was included in the CI, the emission was not regarded as significantly different. The CI calculations were done by using the bootstrapping method "boot" from the R library (boot) and are based on 10,000 replicates. All statistical analyses were done with the software R (R Development Core Team 2005).

\section{Results}

Epichloë Volatiles Analyses of headspace samples focused on three volatile compounds, chokol K, MME, and a trimethylester tentatively identified as MTE. The identification of MTE was done with MS-spectrum comparison from the NIST database and resulted in an $85.3 \%$ match of spectra (Fig. 2). Production of these volatiles are confined to the stroma tissue and could not be detected in samples taken from plant leaves or stems (data not shown). Chokol $\mathrm{K}$ and MME have been shown previously to attract Botanophila flies (Steinebrunner et al. 2008a). The third compound, MTE, is reported from Epichloe for the first time. MTE has not yet been shown to play a role in odor communication; however, due to its similar structure to MME and high abundance in some Epichloë associations, such a function seems likely. The rest of the headspace was made up of various other compounds for which no activity was indicated, and no attempts were made to identify them. 


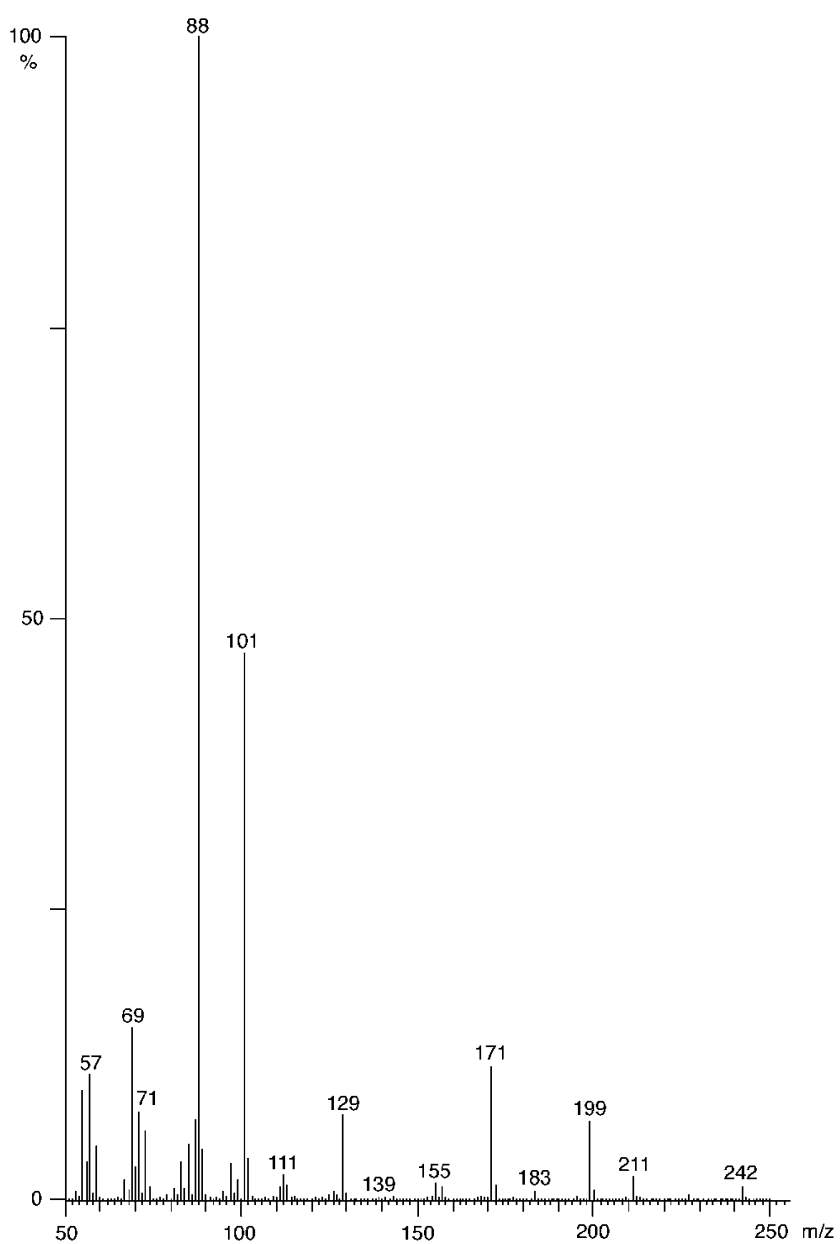

Fig. 2 Mass spectrum of the volatile compound from Epichloë typhina on Puccinellia distans identified as methyl 2,4,8-trimethylundecanoate (MTE) using the NIST database

Relative amounts of chokol K, as determined in headspace samples from fungal stromata, differed among the Epichloë species tested (Kruskal-Wallis test, $\chi=40.86, d f=5, P<$ $0.001)$. Proportion of this compound was highest in Epichloë sylvatica (Es) comprising more than $56 \%$ on average of the total amount in headspace samples (Table 2). In other species, average amounts of chokol $\mathrm{K}$ were lower with $23 \%$ in Epichloë clarkii $(E c)$ and $10 \%$ in E. typhina $(E t)$, while only trace amounts $(<2 \%)$ were found in Epichloë baconii (Eba) and Epichloë bromicola. No chokol $\mathrm{K}$ at all was detected in samples from Epichloë festucae $(E f)$.

Chokol K production differed among the eight host races of E. typhina (Kruskal-Wallis test, $\chi=15.29, d f=7, P=$ $0.018)$. Average emissions were relatively high from $E$. typhina stromata on Anthoxanthum odoratum (Et-Ao), Brachypodium pinnatum (Et-Bp), Phleum pratense (Et$P h p)$, and Poa trivialis $(E t-P t)$ with proportions between $29 \%$ and $52 \%$ (Table 2). Lower emissions $(<2 \%)$ were measured from stromata of E. typhina on Dactylis glomer- ata $(E t-D g)$, Puccinellia distans (Et-Pd), Poa nemoralis $(E t-P n)$, and Poa pratensis $(E t-P p)$. Exceptionally large genotypic differences were observed among accessions of the $E t-B p$ association with regard to the chokol $\mathrm{K}$ production: Five accessions were strong chokol producers ( $49.74 \% \pm 9.27$ SEM, $N=5$ ), while the other two produced only trace amounts $(0.041 \% \pm 0.01, N=2)$.

Proportions of the second volatile MME in headspace samples were also different among Epichloë species (Kruskal-Wallis test, $\chi=24.68, d f=5, P<0.001$ ). Only in samples from $E$. festucae, however, was the average amount high (39\%), whereas in all other associations, no or little MME was found (Table 2). Interestingly, the other two volatiles were not detected in the E. festucae association.

The third volatile MTE showed a similar pattern of uneven distribution among Epichloë species (KruskalWallis test, $\chi=11.35, d f=5, P=0.045$ ). With more than $57 \%$ on average, MTE was the main compound found in samples from E. typhina on Puccinellia distans $(E t-P d)$, where it appears to have replaced the other methyl ester MME. In all other associations, MTE did not exceed 3\% of the total headspace (Table 2).

Principal component analysis (PCA) based on patterns of the three volatile compounds did not discriminate among Epichloë species, except for E. festucae (Fig. 3). E. festucae (4) was separated clearly from the other species owing to its unique pattern with the single dominating volatile MME. The correlation vectors show that a stronger correlation existed between chokol $\mathrm{K}$ and each of the methyl esters than between MME and MTE themselves. The greater length of the chokol $\mathrm{K}$ vector compared to the other vectors indicates that this compound is the best represented variable in the PCA.

Odor profiles of 14 Epichloë accessions representing seven host grasses were evaluated in 2 consecutive years. Mean year differences of volatile emissions were not significantly different from zero at $95 \%$ CI for all three compounds (chokol K, $-4.8 \%$ to $13.53 \%$; MME, $4.5 \%$ to $5.40 \%$; MTE, $-0.1 \%$ to $0.22 \%$ ) suggesting that volatile production by a genotype is consistent and not affected by seasonal variations.

Headspace samples collected from axenic Epichlö̈ cultures in vitro were screened for the three volatile compounds. Of these compounds, only chokol $\mathrm{K}$ was detected, but in small amounts and not in all isolates (Table 2). Chokol K was found in cultures of E. sylvatica (two isolates), E. clarkii (two isolates), and in most $E$. typhina associations (one to two isolates), but not in cultures of E. bromicola (two isolates), E. baconii (two isolates), and E. festucae (two isolates). Generally, genotypes that produced high levels of chokol $\mathrm{K}$ in vivo also produced chokol $\mathrm{K}$ in vitro. In addition, many isolates that 
Table 2 Mean relative amounts (\%) with standard errors $( \pm \mathrm{SE})$ of the volatile compounds chokol $\mathrm{K}$, methyl (Z)-3-methyldodec-2-enoate $(M M E)$, methyl 2,4,8-trimethylundecanoate $(M T E)$, and unidentified diterpenes found in the headspace collected from stromata of different Epichloë associations (in vivo) and from isolates in pure culture (in vitro)

\begin{tabular}{|c|c|c|c|c|c|}
\hline \multirow[t]{3}{*}{ Association $^{\mathrm{a}}$} & \multicolumn{3}{|l|}{ In vivo } & \multicolumn{2}{|l|}{ In vitro } \\
\hline & Chokol K & MME & MTE & Chokol K & Diterpenes \\
\hline & Mean \pm SE & Mean \pm SE & Mean \pm SE & Mean \pm SE & Mean $\pm \mathrm{SE}$ \\
\hline E. bromicola-Be & $0.17 \pm 0.05$ & $0.29 \pm 0.07$ & $0.03 \pm 0.02$ & 0.00 & 0.00 \\
\hline E. baconii (all) & $0.52 \pm 0.25$ & $2.03 \pm 0.88$ & $1.33 \pm 0.93$ & 0.00 & $19.24 \pm 10.06$ \\
\hline$E b a-A t$ & $0.45 \pm 0.29$ & $3.52 \pm 1.01$ & $2.33 \pm 1.51$ & 0.00 & 0.00 \\
\hline$E b a-C v$ & $0.63 \pm 0.52$ & $0.06 \pm 0.06$ & 0.00 & 0.00 & $38.49 \pm 15.00$ \\
\hline E. clarkii-Hl & $23.28 \pm 6.08$ & $0.59 \pm 0.21$ & $0.26 \pm 0.11$ & $0.18 \pm 0.13$ & $10.85 \pm 8.86$ \\
\hline E. festucae-Fr & 0.00 & $38.07 \pm 3.62$ & 0.00 & 0.00 & 0.00 \\
\hline E. sylvatica-Bs & $56.21 \pm 6.79$ & $0.81 \pm 0.45$ & $0.20 \pm 0.19$ & $0.03 \pm 0.02$ & $1.39 \pm 0.39$ \\
\hline E. typhina (all) & $10.20 \pm 2.94$ & $1.47 \pm 0.30$ & $2.67 \pm 1.84$ & $0.02 \pm 0.11$ & $13.05 \pm 3.98$ \\
\hline$E t-A o$ & $29.35 \pm 6.89$ & $1.87 \pm 1.69$ & $0.50 \pm 0.02$ & $1.04 \pm 0.90$ & 0.00 \\
\hline$E t-B p$ & $35.54 \pm 11.18$ & $1.59 \pm 0.57$ & $0.02 \pm 0.02$ & $0.06 \pm 0.06$ & $12.66 \pm 9.68$ \\
\hline$E t-D g$ & $1.06 \pm 0.67$ & $1.97 \pm 0.48$ & $0.13 \pm 0.09$ & $0.03 \pm 0.03$ & $54.73 \pm 28.41$ \\
\hline$E t-P d$ & $0.23 \pm 0.23$ & 0.00 & $57.29 \pm 19.88$ & $0.12 \pm 0.08$ & $19.16 \pm 6.68$ \\
\hline$E t-P h p$ & $45.78 \pm 1.70$ & 0.00 & $4.19 \pm 0.91$ & 0.00 & $7.31 \pm 6.21$ \\
\hline$E t-P n$ & $0.53 \pm 0.27$ & $0.48 \pm 0.22$ & $0.02 \pm 0.01$ & 0.00 & 0.00 \\
\hline$E t-P p$ & $0.15 \pm 0.02$ & $0.31 \pm 0.09$ & $0.30 \pm 0.14$ & $0.32 \pm 0.20$ & 0.00 \\
\hline$E t-P t$ & $51.72 \pm 7.44$ & $0.14 \pm 0.14$ & $1.26 \pm 0.39$ & $0.19 \pm 0.19$ & $8.20 \pm 5.01$ \\
\hline
\end{tabular}

${ }^{\text {a }}$ For host abbreviations, see Table 1.

were found to produce no or only little chokol $\mathrm{K}$ under in vitro conditions produced a range of diterpenes that were not detected in headspace samples from stromata in vivo, notably, E. baconii from Calamagrostis villosa and E. typhina from D. glomerata (Table 2). Others produced neither chokol $\mathrm{K}$ nor diterpenes. These diterpenes are yet unidentified and are referred to in this study as unknown diterpenes (W. Boland, Jena, personal communication).

Phylogenetic Analysis The ML tree attempts to link the phylogeny of Epichloë species with observed odor profiles (Fig. 4). Following Craven et al. (2001), Epichloë species are grouped into two major clades: the "main group of Epichloë species" including E. bromicola, E. baconii, and $E$. festucae (at the base of the phylogram) and the " $E$. typhina complex" including E. typhina (with its host races), E. clarkii, and E. sylvatica. Here, we designate the wellsupported, less basal subclade ( $88 \%$ bootstrap support) within the E. typhina complex as "E. typhina top clade" excluding the more basal $E t-P n$ and $E t-P p$ associations.

High levels of chokol $\mathrm{K}$ with a proportion of more than $10 \%$ in the headspace (levels 3 and 4 ) were found only in Epichloë accessions that were placed in the E. typhina top clade (Fig. 4). An exception was the accessions of $E$. typhina infecting Puccinellia distans from Poland (Et-Pd), whose profiles were dominated by MTE. All other accessions included in the more basal clades produced low amounts or no chokol K (levels 0 and 1). Regarding

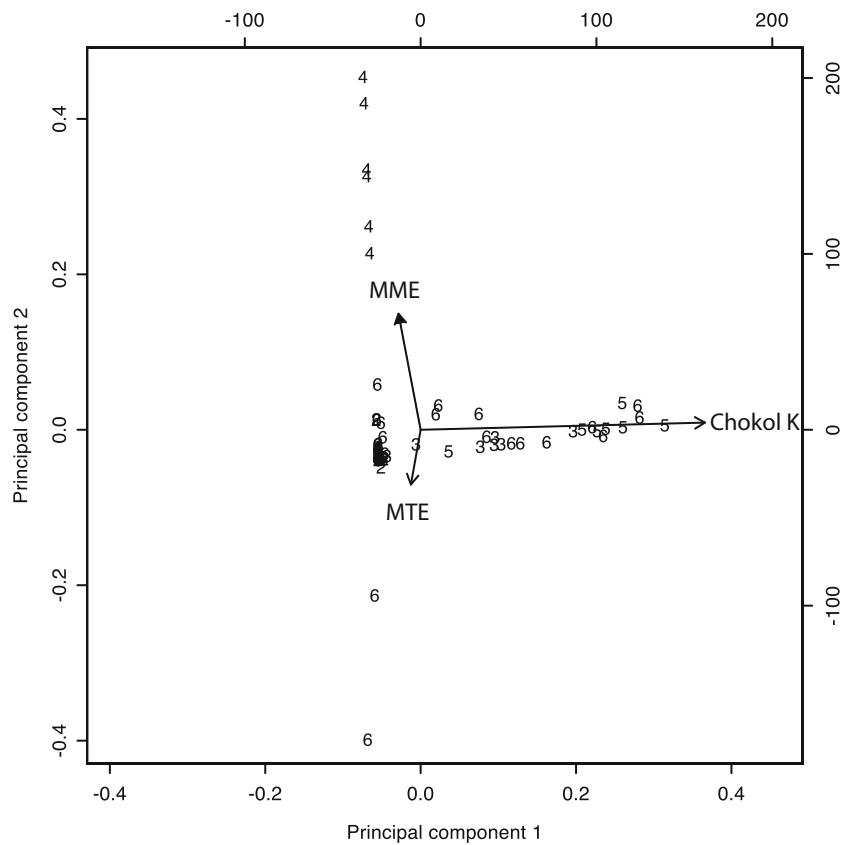

Fig. 3 Principal component analysis of odor compounds chokol K, methyl (Z)-3-methyldodec-2-enoate $(M M E)$, and methyl 2,4,8-trimethylundecanoate $(M T E)$ found in headspace samples from stromata of different Epichloë species (1 E. baconii; 2 E. bromicola; 3 E. clarkii; 4 E. festucae; 5 E. sylvatica; 6 E. typhina). The first two principal component analysis (PCA) factor loadings of the 93 analyzed accessions (genotypes) explain $73 \%$ of the total variance. Correlation arrows give the direction in which the relative amounts of odor compounds from particular genotypes increase. The angles between the arrows correspond to the correlation of the odor variables among each other, while the arrow lengths indicate how well a particular compound is represented in the dataset 
Fig. 4 Maximum likelihood tree (-ln 1600.19) based on fungal tub2 sequences generated by PAUP*4.0b10 and using the $\mathrm{K} 80+\mathrm{G}$ model of evolution. Numbers below the branches are posterior probabilities (based on 1,000 bootstrap replications).

Tabular figures at terminal positions indicate the relative abundance $(0$ absent; $1<1 \% ; 2$ $1-10 \%$; 3 10-30\%; 4 30-70\%; $5>70 \%$ ) of odor compounds [ $C K$ chokol K; MME methyl (Z)-3methyldodec-2-enoate; MTE methyl 2,4,8-

trimethylundecanoate; $D T$ unknown diterpenes] from in vivo and in vitro measurements. The tree is rooted with the outgroup taxon E. brachyelytri from Brachyelytrum erectum $(E b r-B r e)$. Other species and grass host abbreviations are as in Table 1

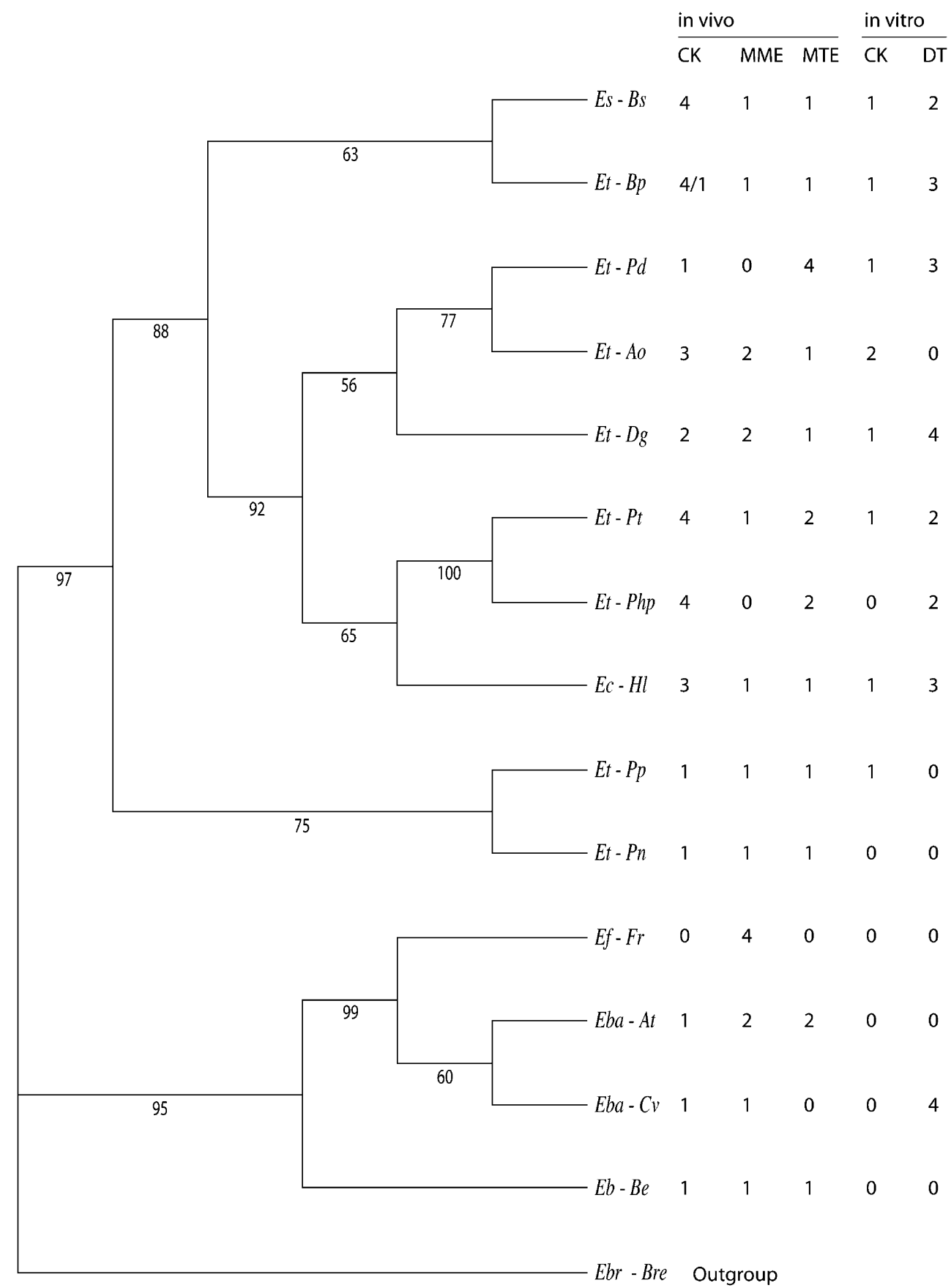

MME, low and moderate levels ( $0-2)$ were found in species of all major clades with one exception. The odor profile of E. festucae of the Epichloë main group was characterized by a high level of MME (level 4) as the dominant volatile. Similarly, the third volatile MTE was present in all major clades at low to moderate levels (0-2) with the exception of association $E t-P d$ mentioned above. The unknown diterpenes (DT) produced in vitro were present only in species of the top clade, again with one exception (Eba-Cv of the main group) and thus mostly occurred in parallel with high levels of chokol $\mathrm{K}$ in vivo.

\section{Discussion}

Evolution of odor profiles in Epichloë fungi may be complex, since host grass and fungal genotypes, the gamete transferring Botanophila flies, and even Epichloë mycoparasites may influence odor evolution. Epichloë species and host races examined in this study exhibited considerable variation in profiles of three volatile compounds, chokol $\mathrm{K}$, MME, and MTE, that have a confirmed or likely role in odor communication. However, profiles overlapped among species, and only few associations exhibited distinct 
profiles. Moreover, there was substantial variation within species, particularly in E. typhina, that naturally infects a range of different host species.

What is the Basis of Odor Variation? The plant material used in our study was from vegetatively propagated accessions, each representing an individual genotypic combination of a host and its systemic endophyte. Among naturally occurring associations, there is a high degree of host specificity, with many Epichloë species apparently forming host races or cryptic species on different hosts (Schardl et al. 2007; Zabalgogeazcoa et al. 2008). Observed variations in odor profiles among and within species may, therefore, be attributed to these more or less stable associations. However, with the sampling scheme used in this study, it is not possible to differentiate between host and fungus contributions to odor variation in Epichloë species that infect multiple hosts, such as E. typhina. To answer this question, a particular endophyte genotype would have to be introduced into different host species experimentally. In closely related Neotyphodium endophytes, investigations on the genetic control of toxic alkaloid secondary metabolites have been made by using experimental infections or reciprocal crosses between infected host plants. These investigations suggested that the ability to produce alkaloids is a property of the fungal genotype, but that the levels of fungal alkaloids may be modified to some extent by plant genotype (Hill et al. 1991; Roylance et al. 1994; Easton et al. 2002), while the influence of environmental factors is usually small (Agee and Hill 1994). In another study that compared endophyte haplotypes in different host genotypes, the host grass significantly influenced alkaloid production (Faeth et al. 2002). In our study, levels of chokol $\mathrm{K}$ and MME collected from the same host-fungus associations in different years remained almost constant between years, suggesting that there is a genetic basis for the observed odor variation and that environmental factors may play a minor role.

In vitro studies may be useful to dissect fungus and plant influences on production of odor compounds. In the present study, only chokol $\mathrm{K}$, but not MME and MTE, were produced under in vitro conditions (Table 2). These findings confirm results from a previous study, which suggested that chokol $\mathrm{K}$ production was genetically encoded in Epichloë, but that for the methylester MME precursory substances of plant origin may be needed (Steinebrunner et al. 2008b). Alternatively, plant-specific signaling may be required for expression of genes involved in the biosynthetic pathway of MME and MTE, as has been shown for the endophyte produced secondary metabolite peramine that protects host plants from insect herbivory (Tanaka et al. 2005) and for an indole-diterpene mycotoxin of Neotyphodium lolii (Young et al. 2006). Moreover, in pure culture when host influences are eliminated, chokol K was detected only in species of the E. typhina complex but was not produced by other Epichloë species of the main clade, suggesting that species depend differently upon plant signaling.

Phylogenetic Analysis Mapping of odor compounds into the corresponding phylogenetic tree of Epichloë taxa did not show a strong phylogenetic signal of odor profiles. However, there was a tendency for Epichlö̈-grass associations in the E. typhina top clade to produce high levels of chokol K (Fig. 4), suggesting that chokol K production was favored early in the speciation of E. typhina. However, weak and strong chokol $\mathrm{K}$ producing genotypes of $E$. typhina were sometimes found within the same association, notably E. typhina on B. pinnatum, while for association $E t-P d$, only low levels of chokol $\mathrm{K}$ were observed. A phylogeny based on odor alone showed a different tree topology, although it was poorly resolved (data not shown). Incongruence between odor phenotypes and the genetic relationship of the producer have been reported for various plants (Williams and Whitten 1999; Barkman 2001; Levin et al. 2003). It has been argued that environmental variation, adaptation to pollinators, and biosynthetic interrelation of compounds would make it difficult to use odor as a character for phylogenetic inference (Levin et al. 2003). Our study did not put the emphasis on validating odor characters for phylogenetic tree reconstruction but on combining DNA sequence and odor information to locate possible evolutionary key events in the phylogeny of Epichloë. Such an event may have been the ability to produce high amounts of chokol $\mathrm{K}$, which occurred at the base of the E. typhina top clade after branching from the more basal E. typhina host races $(E t-P p, E t-P n)$. Chokol K appears to play a role in the attraction of gamete transferring Botanophila flies (Schiestl et al. 2006; Steinebrunner et al. 2008a), and its increased production by E. typhina may have favored host range expansion and evolution of new host races.

Variability of Odor Profiles within and among Species Although there was some structuring with regard to compound levels, odor profiles based on chokol K, MME, and MTE mostly overlapped among species (Fig. 3). Exceptions were the profile of E. festucae that was characterized by the dominance of methyl ester MME and the profile of the Et-Pd association that showed a high level of MTE instead of MME. Thus, Epichloë taxa appear to be little differentiated at the species level with respect to odor profiles, but high variability was observed among genotypes, particularly in associations with E. typhina. Several studies have assessed variability of flower odors among plant species and investigated the use of odor profiles for 
drawing species boundaries (Azuma et al. 1997; Williams and Whitten 1999; Levin et al. 2003; Raguso et al. 2003; Huber et al. 2005). While some of these studies found that each species had its own unique profile (Levin et al. 2003; Huber et al. 2005), others reported a high degree of homoplasy (Williams and Whitten 1999).

Odor emission by Epichloë stromata is thought to be important for the attraction of gamete-transferring Botanophila flies, and thus, patterns or levels of compounds may be selectively adaptive. In a trapping study that used synthetic versions of chokol $\mathrm{K}$ and MME, attraction of Botanophila flies was increased with increasing amounts of these compounds (Steinebrunner et al. 2008a), suggesting that high-odor emission would be advantageous. However, different, taxonomically closely related Botanophila species showed no preference for specific blends that represented different Epichloë taxa, notably E. clarkii with high levels of chokol $\mathrm{K}$ and $E$. festucae with high levels of MME.

The variability in odor profiles observed among genotypes of E. typhina from different accessions may be viewed in the light of Thompson's geographical mosaic theory. This theory predicts that geographic differences in interactions among local selection pressures, genetic drift, and migration create a range of possible outcomes (Thompson 1999). For example, the distinct MTE dominated odor profile in the $E t-P d$ association from Poland may be the result of different selection pressures imposed by the local environment, the Botanophila gametic vectors, or the host plant. Puccinellia distans is a halophyte naturally occurring on marine and inland salines, but infected plants examined in this study originated from highly disturbed anthropogenic habitats in the vicinity of an industrial plant (Lembicz 1998; Olejniczak and Lembicz 2007). Thus, migration of an $E t-P d$ association into this unusual environment and the interaction with the locally occurring Botanophila flies may have resulted in the distinct profile observed for these genotypes. Unfortunately, this hypothesis could not be confirmed because stromaforming plants of Puccinellia are rare outside the industrial sites and were not available. However, local adaptation of the asexual Neotyphodium stage in terms of alkaloid production or herbivore defense has been documented in Achnatherum robustum (Faeth et al. 2006) and in a subarctic fescue (Koh and Hik 2007), suggesting that local adaptation may be a common occurrence in endophyte/ grass symbiota.

Chokol $\mathrm{K}$ is not only involved in odor communication with Botanophila flies but, based on its antifungal properties, may also play a role in protecting Epichloë stromata from mycoparasites (Schiestl et al. 2006; Steinebrunner et al. 2008b). High levels of chokol K typically found in associations of the E. typhina top clade may have also been selectively favored under circumstances of intensive myco- parasite pressure. However, it is not known whether $E$. typhina associations are exposed to such pressure more often compared to the other species and whether mycoparasite resistance is adaptive.

Finally, differences at the Epichloë population level may leave a signature on odor profiles. Depending on whether populations are large or small, selection pressures on volatile production may be enhanced or relaxed. Thus, in small disjunctive populations, Botanophila flies will have to be attracted over a wider distance to guarantee outcrossing, which may select for increased odor production. Moreover, population size differences among species and locations could facilitate genetic drift.

In summary, profiles of three odor compounds produced by Epichloë stromata were often distinctive for particular grass-Epichloë associations but did not discriminate at the species level, except for E. festucae. Although there was some hierarchical structuring regarding levels of chokol $\mathrm{K}$ emission based on the Epichloë phylogeny, odor profiles appeared to be largely dependent on particular Epichloëhost associations. The observed profiles may be the result of complex selective pressures imposed by Botanophila gametic vectors, local environment, and mycoparasites. Future studies should focus on dissecting genetic and environmental factors responsible for the production of odor compounds and on testing host and Epichloë genotype contributions with experimentally manipulated associations by using cross-infections.

Acknowledgments The authors thank Jakov Bolotin for help with MS analyses and Sophie Karrenberg for statistical advice. This research was funded by Swiss National Science Foundation grant no. $3100 \mathrm{~A} 0-101524$.

\section{References}

AgEE, C. S., and HiLL, N. S. 1994. Ergovaline variability in Acremonium-infected tall fescue due to environment and plant genotype. Crop Sci. 34:221-226.

AzUma, H., TOYOTA, M., AsaKaWA, Y., YAMAOKA, R., GarciaFranco, J. G., Dieringer, G., Thien, L. B., and KaWANo, S. 1997. Chemical divergence in floral scents of Magnolia and allied genera (Magnoliaceae). Plant Spec. Biol. 12:69-83.

BARKMAN, T. J. 2001. Character coding of secondary chemical variation for use in phylogenetic analyses. Biochem. Syst. Ecol. 29:1-20.

Bultman, T. L., and Leuchtmann, A. 2003. A test of host specialization by insect vector as a mechanism for reproductive isolation among entomophilous fungal species. Oikos. 103:681687.

Bultman, T. L., White, J. F., Bowdish, T. I., Welch, A. M., and Johnston, J. 1995. Mutualistic transfer of Epichloë-spermatia by Phorbia flies. Mycologia. 87:182-189.

Bultman, T. L., White, J. F., Bowdish, T. I., and Welch, A. M. 1998. A new kind of mutualism between fungi and insects. Mycol. Res. 102:235-238. 
Bultman, T. L., Welch, A. M., Boning, R. A., and Bowdish, T. I. 2000. The cost of mutualism in a fly-fungus interaction. Oecologia. 124:85-90.

Craven, K. D., Hsiau, P. T. W., Leuchtmann, A., Hollin, W., and SCHARDL, C. L. 2001. Multigene phylogeny of Epichloë species, fungal symbionts of grasses. Ann. Missouri Bot. Gard. 88:1434.

Dudareva, N., Negre, F., Nagegowda, D. A., and Orlova, I. 2006. Plant volatiles: Recent advances and future perspectives. Crit. Rev. Plant Sci. 25:417-440.

Easton, H. S., Latch, G. C. M., Tapper, B. A., and Ball, O. J. P. 2002. Ryegrass host genetic control of concentrations of endophyte-derived alkaloids. Crop Sci. 42:51-57.

Faeth, S. H., Bush, L. P., and Sullivan, T. J. 2002. Peramine alkaloid variation in Neotyphodium-infected Arizona fescue: Effects of endophyte and host genotype and environment. $J$. Chem. Ecol. 28:1511-1526.

FAETH, S. H., GARDNER, D. R., HAYes, C. J., Jani, A., Wittlinger, S. K., and JONES, T. A. 2006. Temporal and spatial variation in alkaloid levels in Achnatherum robustum, a native grass infected with the endophyte Neotyphodium. J. Chem. Ecol. 32:307-324.

Grison-PIGE, L., HossaERT-MCKEY, M., GrEeFF, J. M., and BeSSIERE, J. M. 2002. Fig volatile compounds - a first comparative study. Phytochemistry. 61:61-71.

GuevarA, R., RAYNer, A. D. M., and ReYNOLdS, S. E. 2000. Orientation of specialist and generalist fungivorous ciid beetles to host and non-host odors. Physiol. Entomol. 25:288-295.

Hedlund, K., Bengtsson, G., and Rundgren, S. 1995. Fungal odor discrimination in two sympatric species of fungivorous collembolans. Funct. Ecol. 9:869-875.

Hill, N. S., Parrott, W. A., and Pope, D. D. 1991. Ergopeptine alkaloid production by endophytes in a common tall fescue genotype. Crop Sci. 31:1545-1547.

Huber, F. K., Kaiser, R., Sauter, W., and Schiestl, F. P. 2005. Floral scent emission and pollinator attraction in two species of Gymnadenia (Orchidaceae). Oecologia. 142:564-575.

KNUDSEN, J. T. 2002. Variation in floral scent composition within and between populations of Geonoma macrostachys (Arecaceae) in the western Amazon. Am. J. Bot. 89:1772-1778.

KnUdSEn, J. T., ERIKSSON, R., Gershenzon, J., and Stahl, B. 2006. Diversity and distribution of floral scent. Bot. Rev. 72:1-120.

KOH, S., and HIK, D. S. 2007. Herbivory mediates grass-endophyte relationships. Ecology. 88:2752-2757.

Koshino, H., Yoshihara, T., TogiYa, S., Terada, S., Tsukada, S., OKuno, M., Noguchi, A., Sakamura, S., Ichihara, A., ShIMANUKI, T., TAJIMI, A., and SATO, T. 1989. Antifungal compounds from stromata of Epichloë typhina on Phleum pratense, pp. 244-251. Proceedings 31. Symposium on the Chemistry of Natural Products. Sapporo, Japan.

Lembicz, M. 1998. Life history of Puccinellia distans (L.) Parl. (Poaceae) in the colonisation of anthropogenic habitats. Phytocoenosis (N.S.). 10:1-32

Leuchtmann, A. 2003. Taxonomy and diversity of Epichloë endophytes, pp. 169-194, in J. F. White Jr, C. W. Bacon, N. L. HywelJones, and J. W. Spatafora (eds.). Clavicipitalean FungiMarcel Dekker, New York.

LeuchtmanN, A. 2007. Botanophila flies on Epichloë host species in Europe and North America: no evidence for co-evolution. Entomol. Exp. Appl. 123:13-23.

Levin, R. A., Raguso, R. A., and McDade, L. A. 2001. Fragrance chemistry and pollinator affinities in Nyctaginaceae. Phytochemistry. 58:429-440.

Levin, R. A., Mcdade, L. A., and Raguso, R. A. 2003. The systematic utility of floral and vegetative fragrance in two genera of Nyctaginaceae. Syst. Biol. 52:334-351.
OlejNiCZAK, P., and LEMBICZ, M. 2007. Age-specific response of the grass Puccinellia distans to the presence of a fungal endophyte. Oecologia. 152:485-494.

Pellmyr, O., and Thien, L. B. 1986. Insect reproduction and floral fragrances - keys to the evolution of the angiosperms. Taxon. 35:76-85.

Plepys, D., IbARRA, F., Francke, W., and Lofstedt, C. 2002. Odormediated nectar foraging in the silver $\mathrm{Y}$ moth, Autographa gamma (Lepidoptera: Noctuidae): Behavioural and electrophysiological responses to floral volatiles. Oikos. 99:75-82.

PosadA, D., and CRANDALl, K. A. 1998. MODELTEST: Testing the model of DNA substitution. Bioinformatics. 14:817-818.

R Development Core Team. 2005. R: A Language and Environment for Statistical Computing. Vienna.

Raguso, R. A., and RoY, B. A. 1998. 'Floral' scent production by Puccinia rust fungi that mimic flowers. Mol. Ecol. 7:11271136.

Raguso, R. A., Levin, R. A., Foose, S. E., Holmberg, M. W., and MCDADE, L. A. 2003. Fragrance chemistry, nocturnal rhythms and pollination "syndromes" in Nicotiana. Phytochemistry. 63: 265-284.

RaO, S., and BAumanN, D. 2004. The interaction of a Botanophila fly species with an exotic Epichloë fungus in a cultivated grass: fungivore or mutualist? Entomol. Exp. Appl. 112:99-105.

Roylance, J. T., Hill, N. S., and AgeE, C. S. 1994. Ergovaline and peramine production in endophyte-infected tall fescue: Independent regulation and effects of plant and endophyte genotype. $J$. Chem. Ecol. 20:2171-2183.

SAlzmann, C. C., Brown, A., and Schiestl, F. P. 2006. Floral scent emission and pollination syndromes: Evolutionary changes from food to sexual deception. Int. J. Plant Sci. 167:11971204.

Schardl, C. L., Leuchtmann, A., Chung, K. R., Penny, D., and SIEGEL, M. R. 1997. Coevolution by common descent of fungal symbionts (Epichloë spp.) and grass hosts. Mol. Biol. Evol. 14: $133-143$.

Schardl, C. L., Leuchtmann, A., and Mcdonald, B. A. 2007. Relationships of Epichloë typhina isolates from different host grasses, pp. $451-455$, in A. J. Popay, and E. R. Thom (eds.). Proceedings of the 6th International Symposium on Fungal Endophytes of GrassesNew Zealand Grassland Association (Inc.), Dunedin, New Zealand.

Schiestl, F. P., Steinebrunner, F., Schulz, C., von Reuss, S., Francke, W., Weymuth, C., and LeuchtmanN, A. 2006. Evolution of 'pollinator'-attracting signals in fungi. Biol. Lett. 2:401-404.

Schomburg, G. 1990. Gas Chromatography: A Practical Course. Wiley-VCH, Weinheim, New York.

Steinebrunner, F., Twele, R., Francke, W., Leuchtmann, A., and SCHIESTL, F. P. 2008a. Role of odor compounds in the attraction of gamete vectors in endophytic Epichloë fungi. New Phytol. 178:401

Steinebrunner, F., Schiestl, F. P., and Leuchtmann, A. 2008 b. Ecological role of volatiles produced by Epichloë: differences in antifungal toxicity. FEMS Microbiol. Ecol. 64:307-316.

SWOFFORD, D. L. 2003. PAUP: Phylogenetic Analysis Using Parsimony and Other Methods. Sinauer, Sunderland, Massachusetts.

Tanaka, A., Tapper, B. A., Popay, A., Parker, E. J., and Scott, B. 2005. A symbiosis expressed non-ribosomal peptide synthetase from a mutualistic fungal endophyte of perennial ryegrass confers protection to the symbiotum from insect herbivory. Mol. Microbiol. 57:1036-1050.

THOMPSON, J. N. 1999. Specific hypotheses on the geographic mosaic of coevolution. Am. Nat. 153:S1-S14. 
Williams, N. H., and WhitTen, W. M. 1999. Molecular phylogeny and floral fragrances of male euglossine bee-pollinated orchids: A study of Stanhopea (Orchidaceae). Plant Spec. Biol. 14:129136.

Young, C. A., Felitti, S., Shields, K., SPAngenberG, G., Johnson, R. D., BRYAN, G. T., SAIKIA, S., and SCOTT, B. 2006. A complex gene cluster for indole-diterpene biosynthesis in the grass endophyte Neotyphodium lolii. Fungal Genet. Biol. 43:679693.

Zabalgogeazcoa, I., García Ciudad, A., Leuchtmann, A., VÁzQuez de ALdANA, B. R., and García CRiado, B. 2008. Effects of choke disease in the grass Brachypodium phoenicoides. Plant Pathol. published online DOI 10.1111/j.13653059.2007. 01784.x. 